\title{
Bone and joint infections in Oxford: a 10-year retrospective review
}

Movin Abeywickrema ${ }^{1}$, Xinxue Liu ${ }^{1}$, Dominic Kelly ${ }^{1}$, Tim Theologis ${ }^{1}$, Andrew J Pollard ${ }^{1}$, Seilesh Kadambari $^{1}$

${ }^{1}$ Department of Paediatrics, University of Oxford, Level 2, Children's Hospital, John Radcliffe, Headington, Oxford, OX3 9DU

\section{$\underline{\text { Introduction }}$}

Septic arthritis (SA) and osteomyelitis (OM) can present as medical emergencies in children. Typically, antimicrobial treatment for bone and joint infections is between $1-2$ weeks of intravenous treatment followed by a 2-4 week oral antibiotic course.[1,2] However, recent data from a large randomised controlled trial in adults[3] shows that oral therapy is non-inferior to IV therapy. Short IV courses reduce length of hospital stay, support antimicrobial stewardship and improve patient quality of life. We describe the epidemiology of bone and joint infections at the John Radcliffe Hospital, Oxford over the last decade in order to inform improved management.

\section{Methods}

We identified cases of SA and OM from September 2009 to February 2019 using hospital discharge clinical coding data. Inclusion criteria were: i) any child $<16$ years of age ii) with clinical or radiological features typical of SA or OM who were treated as such during their admission and iii) who had $<2$ weeks of symptoms prior to presentation. Clinical data were extracted from electronic patient records. Conventional culture and microscopy were used for microbiological analysis. Samples were not sent for species-specific RT-PCR.

$\underline{\text { Results }}$

\section{Clinical characteristics}

Overall, 343 cases of bone and joint infection were identified over the 10-year surveillance period. 168 cases were excluded (59 cases of insufficient patient information, 48 cases with unconfirmed diagnosis, 8 cases with metalwork in situ, 28 cases of chronic infection, 4 neonatal cases and 21 cases with duration of symptoms $>2$ weeks). 77 cases had OM, 75 cases SA and 23 cases were mixed OM/SA (Table 1).

Data on duration of IV antibiotics were available for 29 SA, 24 OM and 6 mixed OM/SA cases. We found no association between duration of IV antibiotics and length of hospital stay when antibiotic duration was stratified by 1-13 days (median 5.0, 5.0 and 8.0 days for SA, OM and mixed OM/SA respectively), $14-21$ days $(6.5,6.5$ and 11.0 days for $\mathrm{SA}, \mathrm{OM}$ and mixed OM/SA respectively) and $>21$ days (5.5, 8.5 and 7.5 days for $\mathrm{SA}, \mathrm{OM}$ and mixed OM/SA respectively).

Table 1: Clinical characteristics of children with SA, OM and mixed OM/SA. Median results are displayed alongside interquartile ranges and number of cases with data.

\begin{tabular}{|c|c|c|c|}
\hline & $\begin{array}{l}\text { Septic arthritis } \\
{[N=75]}\end{array}$ & Osteomyelitis [N=77] & $\begin{array}{l}\text { Septic arthritis + } \\
\text { Osteomyelitis [ } \mathrm{N}=23]\end{array}$ \\
\hline Median age & $\begin{array}{l}1.45(0.92-4.63) \\
{[n=74]}\end{array}$ & $6.88(1.70-11.57)[n=76]$ & $2.67(0.80-7.00)[n=23]$ \\
\hline Under 5 years & $51(68 \%)$ & $31(40 \%)$ & $14(61 \%)$ \\
\hline Male & $42 / 75(56 \%)$ & $45 / 77(58 \%)$ & $11 / 23(48 \%)$ \\
\hline $\begin{array}{l}\text { Meadianuration of symptoms before } \\
\text { admission (days) }\end{array}$ & $\begin{array}{l}3.00(2.00-5.25) \\
{[n=28]}\end{array}$ & $3.00(2.00-7.00)[n=39]$ & $4.50(2.00-7.75)[n=10]$ \\
\hline
\end{tabular}




\begin{tabular}{|c|c|c|c|c|}
\hline \multicolumn{2}{|l|}{ Median CRP } & $\begin{array}{l}63.90(29.75- \\
123.10)[n=55]\end{array}$ & $25.60(11.30-95.50)[n=53]$ & $58.00(21.75-122.03)[n=16]$ \\
\hline \multicolumn{2}{|l|}{ Median WCC } & $\begin{array}{l}14.90(10.78-18.28) \\
{[n=56]}\end{array}$ & $10.60(7.44-12.60)[n=53]$ & $11.30(8.20-16.60)[n=15]$ \\
\hline \multicolumn{2}{|c|}{ Median duration of admission (days) } & $\begin{array}{l}6.00(4.00-7.25) \\
{[n=40]}\end{array}$ & $5.50(4.75-8.00)[n=39]$ & $9.00(7.00-11.00)[n=8]$ \\
\hline $\begin{array}{l}\text { Site } \\
\text { Upper limb (in } \\
\text { Lower limb (in } \\
\text { Vertebral } \\
\text { Other } \\
\text { Mixed upper/l }\end{array}$ & $\begin{array}{l}\text { uding clavicle) } \\
\text { uding sacroiliac joint) } \\
\text { ver/vertebral }\end{array}$ & $\begin{array}{l}11(15 \%) \\
60(81 \%) \\
1(1 \%) \\
0 \\
2(3 \%)\end{array}$ & $\begin{array}{l}18(23 \%) \\
47(61 \%) \\
3(4 \%) \\
7(9 \%) \\
2(3 \%)\end{array}$ & $\begin{array}{l}7(30 \%) \\
15(65 \%) \\
1(4 \%) \\
0 \\
0\end{array}$ \\
\hline \multirow{3}{*}{$\begin{array}{l}\text { Number } \\
\text { without any } \\
\text { residual } \\
\text { symptoms or } \\
\text { sequelae on } \\
\text { follow-up }\end{array}$} & $\begin{array}{l}\text { 1-13 days of IV } \\
\text { antibiotics }\end{array}$ & $13 / 17(76.5 \%)$ & $8 / 10(80 \%)$ & $0 / 1$ \\
\hline & $\begin{array}{l}\text { 14-20 days of IV } \\
\text { antibiotics }\end{array}$ & $5 / 7(71.4 \%)$ & $10 / 12(83 \%)$ & $4 / 4(100 \%)$ \\
\hline & $\begin{array}{l}21+\text { days of IV } \\
\text { antibiotics }\end{array}$ & $9 / 12(75 \%)$ & $19 / 22(86 \%)$ & $9 / 12(75 \%)$ \\
\hline
\end{tabular}

\section{Microbiology}

\section{Septic arthritis}

$35 / 75$ (47\%) cases had electronically documented blood cultures of which 8 were positive (23\%); other than five cases of Coagulase negative Staphylococci (CONS), there was one positive culture for each of Staphylococcus aureus, Bacillus and Enterobacter cloacae. 53/75 had joint pus cultures with growth of $S$. aureus $(7 / 23,30 \%)$ or $S$. pyogenes $(5 / 23,22 \%)$ being the most common.

\section{Osteomyelitis}

$51 / 77$ (66\%) cases had electronically documented blood cultures of which 18 were positive (35\%); the most common organisms were Staphylococcus aureus (9/18, 50\%) and Group A Streptococcus (4/18, 22\%). 43/77 (56\%) had electronically documented pus cultures. S. aureus was the most common isolate $(6 / 10,60 \%)$.

Table 2: Organisms identified in cases with SA, OM and mixed SA/OM. Median results are displayed alongside interquartile ranges and number of cases with data.

\begin{tabular}{|c|c|c|c|c|c|c|c|}
\hline Microorganism & $\begin{array}{l}\text { Staphylococcus } \\
\text { aureus }[\mathrm{N}=26]\end{array}$ & $\begin{array}{l}\text { Streptococcus } \\
\text { pyogenes/GAS } \\
{[\mathrm{N}=12]}\end{array}$ & $\begin{array}{l}\text { Other gram } \\
\text { positive } \\
\text { cocci* }[\mathrm{N}=13]\end{array}$ & $\begin{array}{l}\text { Kingella } \\
\text { kingae } \\
{[\mathrm{N}=3]}\end{array}$ & $\begin{array}{l}\text { MRSA } \\
{[N=2]}\end{array}$ & $\begin{array}{l}\text { Mixed (2+ } \\
\text { species) } \\
{[\mathrm{N}=12]}\end{array}$ & Other** $[\mathrm{N}=6]$ \\
\hline $\begin{array}{l}\text { Median age of } \\
\text { child }\end{array}$ & $\begin{array}{l}8.85(5.44- \\
14.20)[n=26]\end{array}$ & $\begin{array}{l}4.40(1.75- \\
6.25)[n=12]\end{array}$ & $\begin{array}{l}1.00 \\
(0.75-1.00) \\
{[n=13]}\end{array}$ & $\begin{array}{l}1.00 \\
(0.96- \\
2.50) \\
{[n=3]}\end{array}$ & $\begin{array}{l}7.58 \\
(3.88- \\
11.29) \\
{[n=2]}\end{array}$ & $\begin{array}{l}8.00(3.25- \\
11.25)[n=12]\end{array}$ & $\begin{array}{l}8.50(3.25- \\
11.50)[n=6]\end{array}$ \\
\hline SA (\% of SA) & $6(23 \%)$ & $5(19 \%)$ & $7(27 \%)$ & $1(4 \%)$ & 0 & $4(15 \%)$ & $3(12 \%)$ \\
\hline OM (\% of OM) & $16(44 \%)$ & $5(14 \%)$ & $4(11 \%)$ & 0 & $2(6 \%)$ & $6(17 \%)$ & $3(8 \%)$ \\
\hline $\begin{array}{l}\text { Mixed OM/SA } \\
\text { (\% of mixed } \\
\text { OM/SA) }\end{array}$ & $4(33 \%)$ & $2(17 \%)$ & $2(17 \%)$ & $2(17 \%)$ & 0 & $2(17 \%)$ & 0 \\
\hline CRP & $\begin{array}{l}\mathbf{5 2 . 5 0}(14.40- \\
111.25)[\mathrm{n}=22]\end{array}$ & $\begin{array}{l}148.50 \\
(119.38- \\
166.90)[n=10]\end{array}$ & $\begin{array}{l}48.85(17.65- \\
145.425) \\
{[n=10]}\end{array}$ & $\begin{array}{l}26.00 \\
(16.40- \\
44.90) \\
{[n=3]}\end{array}$ & $\begin{array}{l}33.35 \\
(17.23- \\
49.48) \\
{[n=2]} \\
\end{array}$ & $\begin{array}{l}\text { 47.15 (28.53- } \\
103.48) \\
{[n=10]}\end{array}$ & $\begin{array}{l}11.50(8.00- \\
27.05)[n=3]\end{array}$ \\
\hline WCC & $\begin{array}{l}10.30(7.13- \\
12.60)[n=22]\end{array}$ & 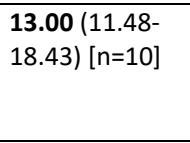 & $\begin{array}{l}\text { 19.20 (11.25- } \\
29)[n=11]\end{array}$ & $\begin{array}{l}\mathbf{1 7 . 1 0} \\
(15.70- \\
18.15) \\
{[n=3]} \\
\end{array}$ & $\begin{array}{l}13.80 \\
(10.70- \\
16.90) \\
{[n=2]} \\
\end{array}$ & $\begin{array}{l}8.25(6.66- \\
10.58)[n=11]\end{array}$ & $\begin{array}{l}6.80(6.80-8.75) \\
{[n=3]}\end{array}$ \\
\hline Surgery & $13 / 24(54 \%)$ & $8 / 11(73 \%)$ & $6 / 11(55 \%)$ & $2 / 3(67 \%)$ & $1 / 2(50 \%)$ & $8 / 12(67 \%)$ & $3 / 4(75 \%)$ \\
\hline $\begin{array}{l}\text { Number } \\
\text { without any } \\
\text { residual } \\
\text { symptoms or }\end{array}$ & $19 / 20(95 \%)$ & $8 / 9(89 \%)$ & $11 / 13(85 \%)$ & $2 / 3(67 \%)$ & $1 / 2(50 \%)$ & $6 / 10(60 \%)$ & $3 / 3(100 \%)$ \\
\hline
\end{tabular}




\begin{tabular}{|c|c|c|c|c|c|c|c|}
\hline $\begin{array}{l}\text { sequelae on } \\
\text { follow-up }\end{array}$ & & & & & & & \\
\hline $\begin{array}{l}\text { Duration of } \\
\text { admission }\end{array}$ & $\begin{array}{l}6.00 \\
(5.00-10.50) \\
{[n=15]}\end{array}$ & $\begin{array}{l}6.00(5.00- \\
7.75)[n=7]\end{array}$ & $\begin{array}{l}5.00(3.50- \\
5.75)[n=6]\end{array}$ & $\begin{array}{l}5.00(3.5- \\
6.5)[n=2]\end{array}$ & $\mathrm{n} / \mathrm{a}$ & $\begin{array}{l}9.50(5.75- \\
20.50)[n=8]\end{array}$ & $\begin{array}{l}5.00(3.5-6.5) \\
{[n=2]}\end{array}$ \\
\hline
\end{tabular}

*Staphylococcus epidermidis, Staphylococcus hominis, Staphylococcus simulans, Streptococcus cristatus, Streptococcus pneumoniae,

Streptococcus parasanguinis, CONS, Group B Streptococcus

**Mycobacterium avium, Salmonella, Bacillus, Cutibacterium, Diphtheroids, Enterobacter cloacae

\section{Discussion}

We showed that SA affects younger children more commonly than OM. S. aureus was the single most common causative agent of both OM and SA which is consistent with a recent national cohort study.[4] We found no clear difference between length of IV therapy and outcome. To our knowledge, there are no robust randomised trials evaluating comparison between IV and PO antimicrobial treatment in childhood OM or SA.

In our centre, over half of all children with bone and joint infections have culture negative disease. Moreover, we detected very low rates of Kingella kingae. Our data highlight the need to utilise molecular assays such as $16 \mathrm{~s}$ PCR to improve pathogen detection.

In conclusion, clinicians should be encouraged to switch to oral antimicrobial therapy following resolution of fever, symptoms and a reduction in inflammatory markers. Studies of oral treatment alone for bone and joint infection in children are warranted.

\section{References}

1 Peltola H, Pääkkönen M, Kallio P, et al. Prospective, randomized trial of 10 days versus 30 days of antimicrobial treatment, including a short-term course of parenteral therapy, for childhood septic arthritis. Clin Infect Dis 2009;48:1201-10. doi:10.1086/597582

2 Saavedra-Lozano J, Falup-Pecurariu O, Faust SN, et al. Bone and Joint Infections. Pediatr Infect Dis J 2017;36:788-99. doi:10.1097/INF.0000000000001635

3 Li H-K, Rombach I, Zambellas R, et al. Oral versus Intravenous Antibiotics for Bone and Joint Infection. N Engl J Med 2019;380:425-36. doi:10.1056/NEJMoa1710926

4 de Graaf H, Sukhtankar P, Arch B, et al. Duration of intravenous antibiotic therapy for children with acute osteomyelitis or septic arthritis: a feasibility study. Health Technol Assess 2017;21:1164. doi:10.3310/hta21480 